\title{
Trans-scale mechanotransductive cascade of biochemical and biomechanical patterning in embryonic development: the light side of the force Tatiana Merle and Emmanuel Farge
}

\begin{abstract}
Embryonic development is made of complex tissue shape changes and cell differentiation tissue patterning. Both types of morphogenetic processes, respectively biomechanical and biochemical in nature, were historically long considered as disconnected. Evidences of the biochemical patterning control of morphogenesis accumulated during the last 3 decades. Recently, new data revealed reversal mechanotransductive feedback demonstrating the strong coupling between embryonic biomechanical and biochemical patterning. Here we will review the findings of the emerging field of mechanotransduction in animal developmental biology and its most recent advancements. We will see how such mechanotransductive cascade of biochemical and mechanical patterning events ensures trans-scale direct cues of coregulation of the microscopic biomolecular activities with the macroscopic morphological patterning. Mechanotransduction regulates many aspects of embryonic development including efficient collective cell behaviour, distant tissues morphogenesis coordination, and the robust coordination of tissue shape morphogenesis with differentiation.
\end{abstract}

\section{Address}

Mechanics and Genetics of Embryonic Development group, Institut Curie, PSL Research University, CNRS, UMR168, Inserm, Marie Curie UnivParis 06, Institut Curie, 11 rue Pierre et Marie Curie, 75005 Paris, France

Corresponding author: Farge, Emmanuel (efarge@curie.fr)

\section{Introduction}

Embryogenesis consists of the development of biomechanical morphology of tissues and biochemical cell differentiation patterns. In the developing embryo, the biomechanical and biochemical patterning were long considered as disconnected. The first was initially thought to be exclusively regulated by Newton's laws of Physics, with tissue growth as a major driving force [1], while the second was subsequently thought to be merely induced by the biochemical cascade based on the interactions between the biomolecules produced by the genome $[2,3]$.

The biochemical regulation of embryonic mechanical patterning by the expression of master differentiation genes was discovered in the 90's, such as mesoderm invagination controlled by the expression of the genes twist and snail in Drosophila melanogaster embryos [4]. In addition, the antero-posterior patterning genes bicoid, nanos and torso-like are required for convergence-extension movements (C\&E) at the onset of Drosophila embryo gastrulation [5]. Similarly, Xwnt-5a was suggested to be involved in C\&E movements in Xenopus embryos [6] and silberblick/wnt11 and wnt5 were found to be necessary for proper C\&E movements in zebrafish embryos [7].

The underlying molecular mechanisms downstream of this regulation are based on a genetic regulation of anisotropies in the intracellular concentration of a molecular motor: Myosin-II (Myo-II) [8]. For instance, the Twist and Snail proteins were found to be required for the medio-apical accumulation of Myo-II in Drosophila embryos. This acto-myosin meshwork constricts mesoderm cell apexes resulting in an apical shrinkage that triggers the mesoderm epithelium invagination [9].

These findings interestingly showed that embryo morphogenesis, that obeys the laws of Newtonian Physics, is the product of the physical conditions and of the biochemical patterns, that both introduce at a given developmental stage the internal forces that will determine the next stage.

Inversely, it has been shown in the 2000s that biochemical developmental patterns at a given stage can also be the product of the biomechanical patterns of the precedent stage through mechanotransduction processes. This introduces at given developmental stages, new biochemical signal inputs of mechanical origin. For instance, in gastrulating Drosophila embryos, mechanical strains developed by $C \& E$ induce beta-catenin ( $\beta$-cat) signalling activation. This activation leads to Twist expression in the anterior endoderm, and is vitally involved in functional anterior mid-gut cell differentiation at larvae stage 
Figure 1

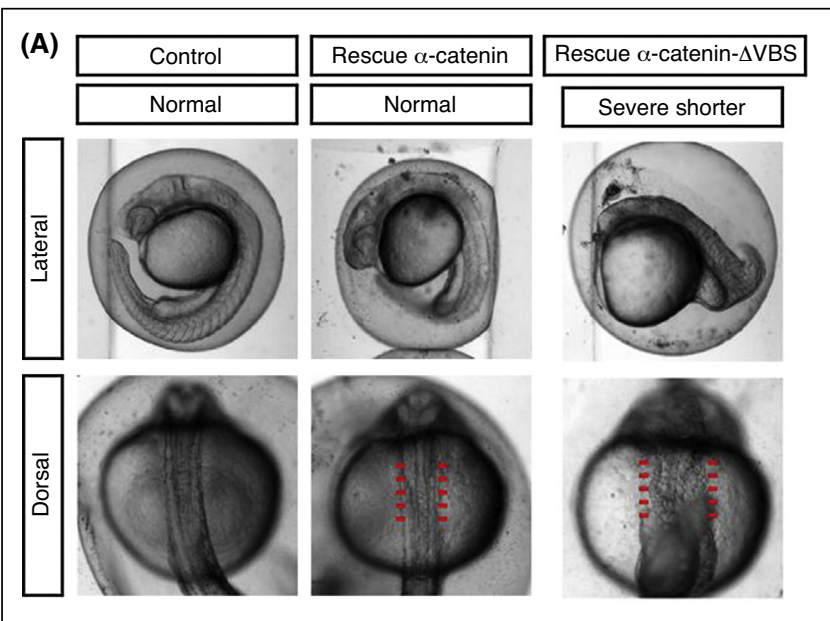

(B)
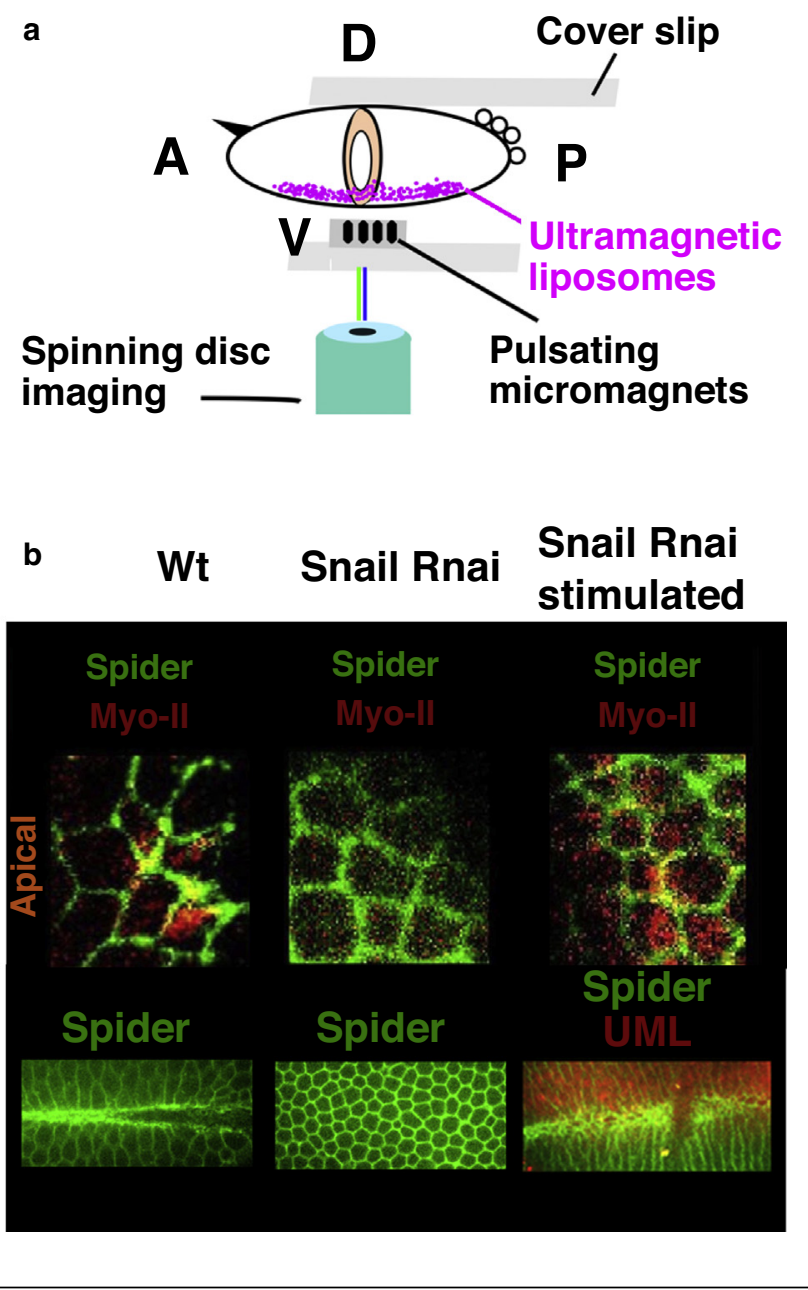

(A) Defect of embryonic convergent-extension morphogenesis development during late epiboly somitogenesis, in the ctnna1 $\mathrm{E}-\alpha$-catenin mutant rescued with the $\mathrm{E}-\alpha$-catenin $\Delta$ VBS lacking the mechanosensitive Vinculin binding site, compared to rescued with the wild-type E- $\alpha$-catenin. (B-a) Quantitative mimicking of soft Snadependent apex pulsations in sna-defective embryos lacking
[10,11]. Similarly, during bone development in mouse embryos, sox 9 is expressed downstream of $\beta$-cat mechanical activation in response to muscle spontaneous activity to prevent bone differentiation in joints [12].

Here we will review the findings and most recent advances in the field reporting the existence of such mechanotransductive cues in animal embryonic development in vivo. We will focus on the mechanotransductive trigger of active morphogenetic biomechanical patterning, and on cell biochemical differentiation and specification patterning. We will describe the physiological functions of such coupling between the developmental biochemical patterning cascade and biomechanical shape development. Based on this trans-scale deterministic coregulation of molecular activities with macroscopic properties, we will present how long-range rapid mechanotransductive cell-cell interactions generate either collective cell behaviour or embryonic morphogenetic movement coordination. We will additionally discuss how it engenders robust coordination between biochemical and biomechanical morphogenesis during embryonic development.

\section{The mechanical trigger of Myo-Il dependent active biomechanical morphogenesis}

Myo-II protein is the molecular motor driving forces in embryonic active morphogenetic movements. Its mechanosensitive behaviour was initially found in cell culture [13]. In vivo, a simple micro-pipet aspiration of Drosophila embryos ectoderm increases the junctional concentration of Myo-II, suggesting a re-enforcement of junctions. Myo-II concentration also increases in ectoderm tissue in response to the C\&E morphogenetic movements of gastrulation in Drosophila embryos [14]. In addition to re-enforcing tissue resistance to deformation, tension also stabilizes Myo-II at the purse-string caused by a wound during Drosophila embryogenesis [15]. The purse string tension increases and generates the wound closure.

The generalisation of such mechanically induced reenforcement of junctions and of tissue integrity in response to embryonic morphogenetic movements was recently observed in early zebrafish embryos. In mutants with central domain-defective $\alpha$-catenin, a domain known to be mechanosensitive in cell culture, the mechanical strains due to gastrulation led to C\&E and embryo elongation defects $\left[16^{\circ}\right]$ (Figure $\left.1 \mathrm{~A}\right)$. In cell

pulsations (here sna RNAi, as well as sna and halo sna mutants -not shown) by magnetic forces generated with pulsatile micro-magnets on the order of the cell size applied on the mesoderm cells injected with ultra-magnetic liposomes, in vivo, (b) rescues the apical accumulation of Myo-II and of the mesoderm invagination from sna-defective embryos lacking apical stabilisation of Myo-II and mesoderm invagination (ventral views). 
culture, such domains mechanically open to effectors such as Vinculin leading to the active re-enforcement of adherens junctions [17]. A similar molecular process was thus proposed to be at work to ensure normal C\&E morphogenesis in response to the strains generated by the earliest embryonic morphogenetic movements $\left[16^{\circ}\right]$. A mechanosensitive maintenance of adherens junctions has also been demonstrated to preserve tissue integrity during Drosophila mesoderm invagination and during $C$-elegans embryo morphogenesis [18,19]. Interestingly, the Yap/Taz mechanosensitive signalling pathway [20] has also been found to be required for the maintenance of the overall shape of the zebrafish embryo. In this case, the Rho GTPase activating protein ARHGAP18 preserves the embryonic tissue stiffness against gravity-induced deformations in a Yap/Taz dependent process [21].

Yet, Myo-II mechanosensitivity is not only involved in the tissue resistance to endogenous morphogenetic movements. It also triggers morphogenetic movements in response to the mechanical strains developed by the morphogenetic movements of the precedent stage. Indeed, the Fog-dependent medio-apical accumulation of Myo-II - that leads to the epithelial mesodermal cells apex constrictions and to mesoderm invagination in Drosophila embryos - has been found to be mechanotransductively induced by the Snail-dependent cell apex pulsations that just precede.

First indicated by mechanical perturbations through indenting the embryo [22], this was demonstrated by the quantitative rescue of sna-dependent pulsations in snail mutants. A network of pulsating micromagnets was applied to snail mutant embryos injected with magnetic liposomes [ $\left.23^{\circ}\right]$. Such physiological mechanical rescue, of pure physical origin, quantitatively mimicked the Snaildependent mechanical strains, and Fog-dependently rescued both the medio-apical accumulation of Myo-II and mesoderm invagination (Figure 1B).

As an underlying molecular mechanism, the sna-dependent apex pulsations were found to mechanically inhibit Fog endocytosis and to enhance Fog exocytosis, presumably by membrane flattening and tension increase, thereby triggering the Rho downstream pathway leading to medio-apical accumulation of Myo-II [22]. Interestingly, the existence of a defect of endocytosis in the mesoderm versus the ectoderm was recently corroborated in early gastrulating Drosophila embryos [24].

In addition to mechanotransductive cues, the shape of the embryo was found to determine the shape of the actomyosin meshwork [25].

Furthermore, the mechanical strains developed by mesoderm invagination stretches the posterior pole of the embryo, in which Fog is also expressed. As a consequence, this leads to the Fog-dependent mechanotransductive activation of apical Myo-II accumulation and to the initiation of the posterior endoderm invagination $\left[23^{\circ}\right]$. This process, in turn, mechanically participates in the morphogenetic movement of C\&E by anteroposterior cell elongation [26,27]. And this C\&E morphogenetic movement also positively feedbacks into apical Myo-II accumulation in the posterior endoderm and robustly participate together with mesoderm invagination in the initiation of the posterior endoderm invagination $\left[23^{\circ}\right]$. Thus, the mechanosensitive behaviour of Myo-II is at the node of an active self-induced biomechanical cascade of morphogenetic movements. Mechanotransductive feedback processes are also involved in organogenesis. The mechanical strains developed by contractile apoptotic cells were found to enhance junctional apicobasal Myo-II concentration in neighbouring cells, thereby leading to tissue folding in Drosophila leg joint formation [28]. Cells can also stock geometrical surrounding information via biochemical cues to control their own geometrical properties. In the Drosophila pupal epithelium, through the recruitment of Mud proteins, tricellular junctions (TCJs) orient cell division via the astral microtubules by pulling on the mitotic spindle [29 $9^{\bullet \bullet}$. Mechanotransduction is also involved in plant development. Meristem shaping and coordinated cell growth are regulated by mechano-sensitive microtubule reorganisation via Katanin [30,31].

An active biomechanical patterning cascade is thus at work during embryonic development, adding to the wellknown biochemical inductive cascade of patterning gene expression.

Moreover, as we will see, the mechanical strains developed by the biomechanical cascade can also participate, in turn, to determine the biochemical patterning cascade.

\section{The mechanical regulation of biochemical patterning}

Myo-II is by far not the only biomolecule whose behaviour is mechanosensitive. Transcription or co-transcription factor activation can also be strain dependent. For instance, the transcription factor NF-KB translocates into the nucleus in response to mechanical deformation in endothelial cells in culture leading to PDGF growth factor expression [32]. In zebrafish, laminar shear stress generated by blood flow induces Yap nuclear translocation. This transcription factor then stimulates endothelial cell division and contributes to vessel maintenance [33]. And Smad1 is one of the first transcription factors shown to be mechanotransductively involved in cell differentiation by triggering myoblast-osteoblast transdifferentiation [34].

In vivo, the $\beta$-cat protein is responsible for the mechanical induction of the twist mesodermal gene expression in the 
early drosophila ectoderm submitted to external uni-axial global deformation [10]. The mechanosensitivity of $\beta$-cat was found to vitally trigger the mechanical induction of Twist expression in the anterior endoderm. $\beta$-Cat activates the downstream differentiation of the anterior midgut following its mechanically induced partial release from the cell junctions and its transfer from the cytoplasm to the nucleus in response to C\&E compression during gastrulation. To perturb mechanical strains in epithelium and inhibit the anterior endoderm compression within the wild-type genetic background (WT), compressing tissues were ablated with a two photons laser. To rescue physiological compressions in the WT, a magnetic field gradient was used to micromanipulate the neighbouring tissues loaded with super-paramagnetic nano-particles [11].

The $\beta$-cat mechanosensitivity is also an important mechanism to stimulate and trigger mesodermal genes expression in the Drosophila (Twist) and zebrafish (brachyury) embryos mesoderm, in response to its invagination and to epiboly initiation, respectively. Under mechanical tensions, Src42A phosphorylates the Y654- $\beta$-cat major site of interaction with E-cadherins (E-cad) leading to the release of a pool of $\beta$-cat from the junctions to the cytoplasm and the nucleus [35]. Mechanical induction might also be involved in brachyury expression during Nematostella gastrulation [36].

Interestingly in pathological contexts, $\beta$-cat mechanical activation, leading to the expression of tumorous target genes like cyclin D1, myc and zeb-1 in strained healthy tissues, is also involved in tumor progression due to skin and breast tumours stiffness and colon tumor growth pressure in vivo $[37,38]$. We also can notice that treatments activating the $\beta$-cat pathway with mechanical vibrations in adipocytes, repressing the adipogenic Glycogene Synthase, are developed to fight against obesity [39].

$\beta$-cat mechanosensitivity was recently found to be physiologically involved in several distinct developmental processes. In particular, it was proposed to initiate feather follicle differentiation during chicken development. Indeed, in response to epidermis buckling under MyoII contractile tension, $\beta$-cat becomes phosphorylated on Y654 and translocates into the nucleus of bent cell domains. When Myo-II contractile activity was blocked with blebbistatin, no tissue buckling was observed. As a result, $\beta$-cat activation and the expression of its target gene $b m p 2$, known to be upstream of follicle cell differentiation, were inhibited $\left[40^{\circ}\right.$ ] (Figure $2 \mathrm{~A}$ ).

In addition to $\beta$-cat, phosphorylation of the mechanosensitive Yap factor was also recently found to regulate the earliest differentiation of inner-cell mass of pre-implantation mouse embryos, during cell engulfment morphogenesis. This follows the first asymmetric division after fertilization, and was proposed to be the consequence of the high acto-myosin contraction of the cell engulfed by the less contractile one $\left[41^{\circ}\right]$. The inhibition of the MyoII contractile activity with blebblistatin prevents cell engulfment. As a result, in a doublet, both cells express inner cell-like markers, that is high levels of cytoplasmic phosphorylated-Yap and low levels of $\mathrm{Cdx} 2$ expression (Figure 2B).

We saw above how internal morphogenetic forces can regulate tissue developmental biochemical patterning. Interestingly, mechanical strains that are external to tissues can also influence biochemical development.

For instance, mechanical constraints applied by the uterus on the growing mouse embryo lead to the mechanical induction of Cer1 expression in its distal endoderm. This protein is involved in the determination of the antero-posterior axis of mouse embryonic development [42].

In late stages of zebrafish embryos development, the oscillatory shear stress induced by the blood flow stimulates the expression of the endocardial flow-responsive klf2a gene. This gene was found to control the fibronectin synthesis that initiates cardiac valve development through the canonical Wnt/ $\beta$-cat pathway [43,44].

In mouse embryos, the oscillatory shear stress of the lymphatic fluid enhances the $\beta$-cat signalling pathway in endothelial cells, and the expression of target genes, such as FOXC2, that regulates lymphatic vasculature development [45].

\section{The integrative physiological functions of trans-scale mechanical and mechanotransductive cues in development}

Predicting the macroscopic behaviours of living systems based on their molecular activities through an integrative reductionist approach from one scale to another remains an active field of biology. The challenge lies in untangling the complex interactions through different scales. In contrast, mechanical strains consist of a very simple cue, as it crosses all scales, thereby directly coupling the molecular microscopic with the macroscopic patterning scale. Indeed, as we saw, the Myo-II activity, which is biochemically patterned, generates mechanical strains leading to macroscopic biomechanical morphogenetic movements. These morphogenetic movements in turn mechanotransductively modulate the activity of Myo-II at the molecular level, which triggers other active morphogenetic movements. Macroscopic strains additionally regulate the biochemical activities leading to (co-)transcription factor nuclear translocation at the molecular level, thereby modulating developmental gene expression. 


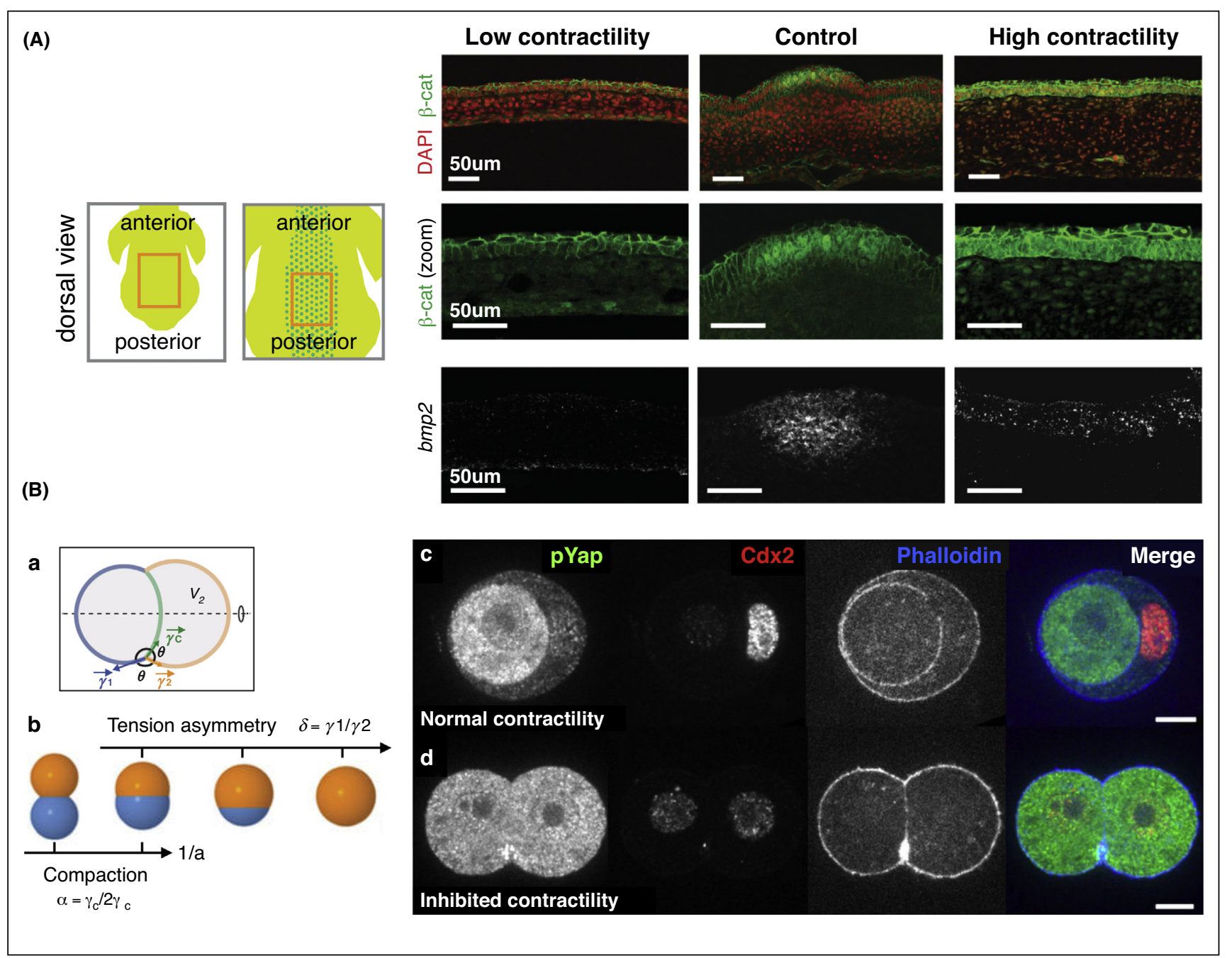

(A) Contractility triggers $\beta$-catenin nuclearisation in the forming primordium of early chicken embryos and is upstream of the primordia gene expression program. Contractility is low on the left due to blebbistatin treatment ( $25 \mu \mathrm{M} \mathrm{Bb})$ and high on the right due to high substrate stiffness without blebbistatin treatment ( $25 \mathrm{nM} \mathrm{CA}$ ) compared to control conditions on the centre. $\beta$-catenin localization (IF), and bmp2 expression (FISH) depends on the contractility and on the buckling deformation of the sample. (B) First morphogenetic movement of cell internalisation in mouse embryos mechanically depends on the cell contractility asymmetry and leads to asymmetric differentiation. (a) Diagram of cell doublet. $\gamma 1$, $\gamma 2$ and $\gamma \mathrm{c}$ are the surface tensions of the two cells and the contact. (b) Numerical simulation showing the equilibrium state of a doublet from a 16-cellstage mouse embryo. The first cell fate depends on the equilibrium state of the doublet. (c,d) Immunostaining of control (25 $\mu \mathrm{M}$ of non-efficient $\mathrm{Bb})$ and inhibited by blebbistatin $(25 \mu \mathrm{M} \mathrm{Bb})$ doublets showing $\mathrm{p}$-Yap, the Cdx2 outer layer cells expression and phalloidin.

In other words, in embryonic patterning, the mechanosensitivity of the biochemical and active biomechanical components allows mechanical ${ }^{\text {cues }}$ to be privileged mode of direct interaction between the biochemically active and reactive microscopic molecular regulators of embryogenesis and the macroscopic morphological phenotype of the embryo $[46,47]$.

Among the physiological functions of such mechanobiochemical cascade that underlie embryogenesis, we can notice integrative rapid and long-range cell-cell interactions. For instance, in the Drosophila embryo, line tensions of acto-myosin cables robustly maintain differentiation boundaries across tissues [48,49]. Also, collective cell apex constrictions, coordinated across the mesoderm tissue by rapid and long-range mechanotransductive cell-cell interactions, are required for tissue invagination at gastrulation $\left[23^{\circ}\right]$.

Physiological functions also reveal a robust coordination between biomechanical and biochemical patterning. For instance, the mechanical induction of Twist expression ensures that only the internalized tissue at gastrulation will maintain Twist expression, and will in fine 
differentiate into mesodermal internal organs [35]. Similarly in early mouse embryos only the not-ingulfed cells express the Cxd2 gene responsible for the trophectoderm identity, and will not develop as an embryonic tissue $\left[41^{\bullet}\right]$.

Such mechanical cues were additionally found, and proposed, to mediate long-range coordination of biomechanical and biochemical patterning, throughout the whole embryo. For instance, ventral mesoderm invagination triggers the initiation of posterior endoderm invagination within the 2 min time scale, thereby coordinating mesoderm with endoderm invagination at gastrulation [23 $3^{\circ}$. In addition, germ-band extension mediates both the anterior endoderm specification - through $\beta$-cat activation - and the posterior endoderm invagination — through the Fog/ MyoII pathway —, thereby synchronising two processes 100 cells $(500 \mu \mathrm{m})$ apart $\left[11,23^{\circ}\right]$. These effects may be of particular importance under conditions in which the dynamics and complex topology of the embryonic tissues prevent the establishment of the long-range morphogen gradients that are efficient at earliest stages where cells are arranged in simpler, static geometrical patterns.

And indeed, integrating both the macroscopic biomechanical and microscopic biochemical patterns through such reciprocal trans-scale mechanical and mechanotransductive interaction, explains

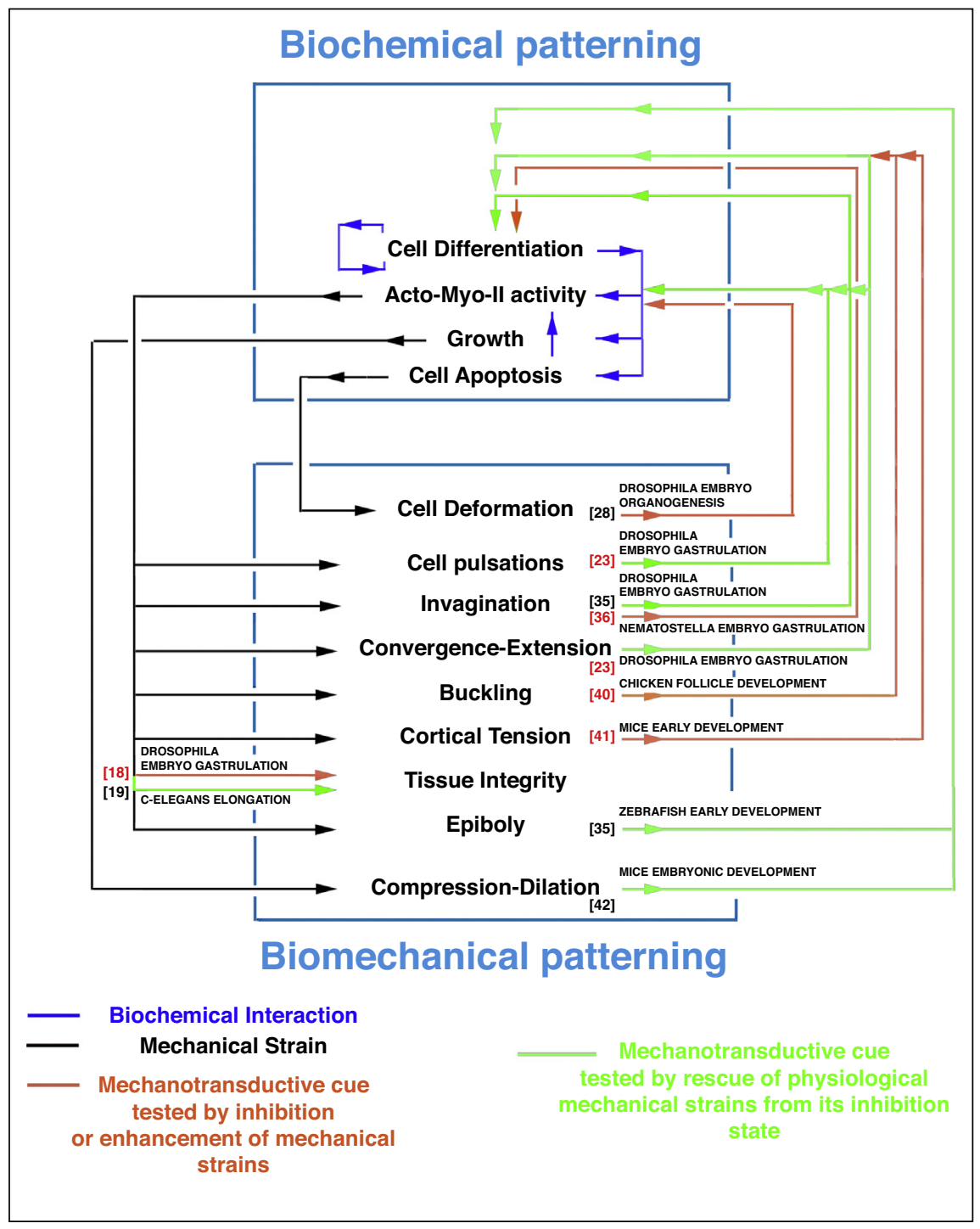

Synthesis of the findings of mechanotransdutive cues found as involved in embryonic development (green and brown arrows), within the general context of biochemical interactions (arrows in blue) and of the biochemical regulation of biomechanical patterning (arrows in black). Most recent findings of the two last years are referred in red. 
embryogenetic processes as important as gastrulation, initial tissue differentiation, maintenance of patterning boundaries, coordination of morphogenetic movements and the coordination of biomechanical/biochemical patterning (Figure 3).

\section{Conflict of interest statement}

\author{
Nothing declared.
}

\section{Acknowledgements}

The 'Mechanics and Genetics of Embryonic and Tumor Development' lab is currently funded by the FRM (Grant \# DEQ20150331702), the ANR (Grant \# 16CE14002801), and the PSL-IRIS-OCAV (Grant \# ANR1-10IDEX-0001-02 PSL). Tatiana Merle is funded by the ED-PIF (doctoral funding 1767/2014) and the FRM (Grant \# FDT20170436944).

\section{References and recommended reading}

Papers of particular interest, published within the period of review, have been highlighted as:

- of special interest

-. of outstanding interest

1. Thomson DAW: On Growth and Form. 1917

2. Lewis EB: A gene complex controlling segmentation in Drosophila. Nature 1978, 276:565-570.

3. Nusslein-Volhard C, Wieschaus E: Mutations affecting segment number and polarity in Drosophila. Nature 1980, 287:795-801.

4. Leptin M: Twist and snail as positive and negative regulators during Drosophila mesoderm development. Genes Dev 1991 5:1568-1576.

5. Irvine KD, Wieschaus E: Cell intercalation during Drosophila germband extension and its regulation by pair-rule segmentation genes. Development 1994, 120:827-841.

6. Moon RT, Campbell RM, Christian JL, McGrew LL, Shih J, Fraser S: Xwnt-5A: a maternal Wnt that affects morphogenetic movements after overexpression in embryos of Xenopus laevis. Development 1993, 119:97-111.

7. Heisenberg CP, Tada M, Rauch GJ, Saude L, Concha ML, Geisler R, Stemple DL, Smith JC, Wilson SW: Silberblick/Wnt11 mediates convergent extension movements during zebrafish gastrulation. Nature 2000, 405:76-81.

8. Young PE, Pesacreta TC, Kiehart DP: Dynamic changes in the distribution of cytoplasmic myosin during Drosophila embryogenesis. Development 1991, 111:1-14.

9. Dawes-Hoang RE, Parmar KM, Christiansen AE, Phelps CB, Brand $\mathrm{AH}$ : Wieschaus EF: folded gastrulation, cell shape change and the control of myosin localization. Development 2005, 132:4165-4178.

10. Farge $\mathrm{E}:$ Mechanical induction of twist in the Drosophila foregut/stomodeal primordium. Curr Biol 2003, 13:1365-1377.

11. Desprat N, Supatto W, Pouille P-A, Beaurepaire E, Farge E: Tissue deformation modulates twist expression to determine anterior midgut differentiation in Drosophila embryos. Dev Cell 2008, 15:470-477.

12. Kahn J, Shwartz Y, Blitz E, Krief S, Sharir A, Breitel DA, Rattenbach R, Relaix F, Maire P, Rountree RB et al.: Muscle contraction is necessary to maintain joint progenitor cell fate Dev Cell 2009, 16:734-743.

13. Effler JC, Kee YS, Berk JM, Tran MN, Iglesias PA, Robinson DN: Mitosis-specific mechanosensing and contractile-protein redistribution control cell shape. Curr Biol 2006, 16:1962-1967.

14. Fernandez-Gonzalez R, Simoes Sde M, Roper JC, Eaton S, Zallen JA: Myosin II dynamics are regulated by tension in intercalating cells. Dev Cell 2009, 17:736-743.
15. Kobb AB, Zulueta-Coarasa T, Fernandez-Gonzalez R: Tension regulates myosin dynamics during Drosophila embryonic wound repair. J Cell Sci 2017, 130:689-696.

16. Han MK, Hoijman E, Noel E, Garric L, Bakkers J, de Rooij J: - alphaE-catenin-dependent mechanotransduction is essentia for proper convergent extension in zebrafish. Biol Open 2016 , 5:1461-1472.

Here, Han et al. confirmed the key role of $\alpha$-cat mechanosensitivity in the correct regulation of C\&E movements. In $\alpha$-cat mutants, they rescued cadherin adhesion, but not $\alpha$-cat mechanosensitive function, by injecting $\alpha$-cat mRNAs with a deficient mechanotransductive site: the Vinculin interacting site domain. In cell culture, this domain mechanically opens to Vinculin when $\alpha$-cat is stretched leading to adherens junction re-enforcement. Interestingly, mRNA injections led to defects in the polarity of mesoderm cell movements towards the dorsal pole of the embryo, possibly due to adherens junctions remodeling/stabilization defects. This generated defects in the C\&E movement required for proper embryonic morphogenesis, including embryo elongation, demonstrating the necessity for this mechanotransductive site in zebrafish development.

17. Yao M, Qiu W, Liu R, Efremov AK, Cong P, Seddiki R, Payre M, Lim CT, Ladoux B, Mege RM et al.: Force-dependent conformational switch of alpha-catenin controls vinculin binding. Nat Commun 2014, 5:4525.

18. Weng M, Wieschaus E: Myosin-dependent remodeling of adherens junctions protects junctions from Snail-dependent disassembly. J Cell Biol 2016, 212:219-229.

19. Zhang $\mathrm{H}$, Landmann $\mathrm{F}$, Zahreddine $\mathrm{H}$, Rodriguez D, Koch M, Labouesse M: A tension-induced mechanotransduction pathway promotes epithelial morphogenesis. Nature 2011, 471:99-103.

20. Dupont S, Morsut L, Aragona M, Enzo E, Giulitti S, Cordenonsi M Zanconato F, Le Digabel J, Forcato M, Bicciato S et al.: Role of YAP/TAZ in mechanotransduction. Nature 2011, 474:179-183.

21. Porazinski S, Wang H, Asaoka Y, Behrndt M, Miyamoto T, Morita H, Hata S, Sasaki T, Krens SF, Osada Y et al.: YAP is essential for tissue tension to ensure vertebrate 3D body shape. Nature 2015, 521:217-221.

22. Pouille PA, Ahmadi P, Brunet AC, Farge E: Mechanical signals trigger myosin II redistribution and mesoderm invagination in Drosophila embryos. Sci Signal 2009, 2:ra16.

23. Mitrossilis D, Roper JC, Le Roy D, Driquez B, Michel A, Menager C,

- Shaw G, Le Denmat S, Ranno L, Dumas-Bouchiat F et al.: Mechanotransductive cascade of Myo-II-dependent mesoderm and endoderm invaginations in embryo gastrulation. Nat Commun 2017, 8:13883.

Here, to rescue thesnail-dependent soft fluctuations, Mitrossilis et al used a network of oscillating micromagnets in front of the mesoderm of snail mutant embryos injected with ultra-magnetic liposomes. Importantly, this network could generate pulsatile magnetic forces and restore dynamically the missing forces in vivo at the cell-scale resolution. Mitrossilis et al. rescued both Myo-II apical accumulation and mesoderm invagination demonstrating that Fog-dependent mechanotransductive cues trigger gastrulation in response to snail-dependent apex pulsations in vivo. This demonstrated that gastrulation is mechanotransductively triggered by cell apex size fluctuations in Drosophila embryos.

24. Jha A, van Zanten TS, Philippe JM, Mayor S, Lecuit T: Quantitative control of GPCR organization and signaling by endocytosis in epithelial morphogenesis. Curr Biol 2018.

25. Chanet S, Miller CJ, Vaishnav ED, Ermentrout B, Davidson LA, Martin AC: Actomyosin meshwork mechanosensing enables tissue shape to orient cell force. Nat Commun 2017, 8:15014.

26. Lye CM, Blanchard GB, Naylor HW, Muresan L, Huisken J, Adams RJ, Sanson B: Mechanical coupling between endoderm invagination and axis extension in Drosophila. PLOS Biol 2015, $13 \mathrm{e} 1002292$.

27. Collinet C, Rauzi M, Lenne PF, Lecuit T: Local and tissue-scale forces drive oriented junction growth during tissue extension. Nat Cell Biol 2015, 17:1247-1258.

28. Monier B, Gettings M, Gay G, Mangeat T, Schott S, Guarner A, Suzanne M: Apico-basal forces exerted by apoptotic cells drive epithelium folding. Nature 2015, 518:245-248. 
29. Bosveld F, Markova O, Guirao B, Martin C, Wang Z, Pierre A,

-• Balakireva M, Gaugue I, Ainslie A, Christophorou N et al.: Epithelial tricellular junctions act as interphase cell shape sensors to orient mitosis. Nature 2016, 530:495-498.

InDrosophila pupal epithelium, cells round up during mitosis and therefore loose their interphase shape anisotropy. In these conditions, Bosveld et al. asked how cells can achieve the Herwig's rule that stipulates that cells will divide along their long-axis direction. They showed that tricellular junctions (TCJs) recruit the Dynein-associated protein Mud to exert forces on the mitotic spindle and control its position during mitosis. Using laser ablation, they could show that Mud pulls forces via the astra microtubules. Remarkably, the predictions of the associated Mud-mode were significantly better that the former Shape-model ones. By showing how TCJs play a major role in orienting cell divisions in Drosophila embryo epithelium, this study describes how cells can stock geometrical surrounding information via biochemical cues and control their own geometrical properties.

30. Uyttewaal M, Burian A, Alim K, Landrein B, Borowska-Wykret D, Dedieu A, Peaucelle A, Ludynia M, Traas J, Boudaoud A et al.: Mechanical stress acts via katanin to amplify differences in growth rate between adjacent cells in Arabidopsis. Cell 2012 149:439-451.

31. Verger S, Long $\mathrm{Y}$, Boudaoud A, Hamant O: A tension-adhesion feedback loop in plant epidermis. Elife 2018, 7.

32. Hay DC, Beers C, Cameron V, Thomson L, Flitney FW, Hay RT: Activation of NF-kappaB nuclear transcription factor by flow in human endothelial cells. Biochim Biophys Acta 2003, 1642:3344.

33. Nakajima $\mathrm{H}$, Yamamoto $\mathrm{K}$, Agarwala $\mathrm{S}$, Terai $\mathrm{K}$, Fukui $\mathrm{H}$, Fukuhara S, Ando K, Miyazaki T, Yokota Y, Schmelzer E et al.: Flow-dependent endothelial YAP regulation contributes to vessel maintenance. Dev Cell 2017, 40:523-536 e526.

34. Rauch C, Brunet AC, Deleule J, Farge E: C2C12 myoblast/ osteoblast transdifferentiation steps enhanced by epigenetic inhibition of BMP2 endocytosis. Am J Physiol Cell Physiol 2002, 283:C235-243.

35. Brunet T, Bouclet A, Ahmadi P, Mitrossilis D, Driquez B, Brunet AC, Henry L, Serman F, Béalle G, Ménager C et al.: Evolutionary conservation of early mesoderm specification by mechanotransduction in Bilateria. Nat Commun 2013, 4.

36. Pukhlyakova E, Aman AJ, Elsayad K, Technau U: beta-Catenindependent mechanotransduction dates back to the common ancestor of Cnidaria and Bilateria. Proc Natl Acad Sci U S A 2018.

37. Fernandez-Sanchez ME, Barbier S, Whitehead J, Bealle G, Michel A, Latorre-Ossa H, Rey C, Fouassier L, Claperon A, Brulle L et al.: Mechanical induction of the tumorigenic beta-catenin pathway by tumour growth pressure. Nature 2015, 523:92-95.

38. Samuel MS, Lopez JI, McGhee EJ, Croft DR, Strachan D, Timpson P, Munro J, Schroder E, Zhou J, Brunton VG et al.: Actomyosin-mediated cellular tension drives increased tissue stiffness and beta-catenin activation to induce epidermal hyperplasia and tumor growth. Cancer Cell 2011, 19:776-791.

39. Shoham N, Gefen A: Mechanotransduction in adipocytes. $J$ Biomech 2012, 45:1-8.

40. Shyer AE, Rodrigues AR, Schroeder GG, Kassianidou E, Kumar S,

- Harland RM: Emergent cellular self-organization and mechanosensation initiate follicle pattern in the avian skin. Science 2017, 357:811-815.

Shyer et al. used nascent skin of chicken embryos to study the follicle formation. They importantly propose that the self-cellular aggregation, giving rise to tissue compaction and buckling, triggers the $\beta$-catenin activation and initiates the downstream follicle genebmp2. Interestingly, they could demonstrate that $\beta$-cat is activated through the phosphorylation of its highly conserved Y654 site [50]. These findings are consistent with the observations in Drosophila and zebrafish embryos [35]. By blocking acto-myosin tension with blebbistatin, the authors blocked both the follicle tissue buckling, and the activation of the $\beta$-cat pathway with the expression of its target gene bmp2 that initiates follicle differentiation and formation. By increasing the stiffness of the cell culture substrate, the authors prevented buckling and presumably increased the mean tension overall the tissue, consistently leading to the ectopic activation of the $\beta$-cat pathway and of the expression of its target gene bmp2.

41. Maitre JL, Turlier H, Illukkumbura R, Eismann B, Niwayama R,

- Nedelec F, Hiiragi T: Asymmetric division of contractile domains couples cell positioning and fate specification. Nature 2016, 536:344-348.

Maître et al. proposed that the first morphogenetic movements - here engulfment - of the mouse vertebrate embryo is crucial for primary differentiation between embryonic cell mass and cell layer external tissues. The differentiation of the inner cell mass, that will eventually lead to the embryonic tissue in contrast to the external future cell layer, is importantly proposed to be mechanically regulated through the signalling of the Yap mechanosensitive factor. By blocking acto-myosin contractibility with blebbistatin, the authors blocked cell engulfment, Yap signalling pathway activation and the expression of its target gene Cdx2 marker of outer cell differentiation. The rescue of outer-cell-like fate with physical tools mimicking physiological cortical tension in blebbistatin-treated embryos would definitively demonstrate that such fate specification in mouse embryos is mechanically induced.

42. Hiramatsu R, Matsuoka T, Kimura-Yoshida C, Han SW Mochida K, Adachi T, Takayama S, Matsuo I: Externa mechanical cues trigger the establishment of the anteriorposterior axis in early mouse embryos. Dev Cell 2013, 27:131144.

43. Steed E, Faggianelli N, Roth S, Ramspacher C, Concordet JP Vermot J: klf2a couples mechanotransduction and zebrafish valve morphogenesis through fibronectin synthesis. Nat Commun 2016, 7:11646.

44. Goddard LM, Duchemin AL, Ramalingan $\mathrm{H}$, Wu B, Chen M, Bamezai S, Yang J, Li L, Morley MP, Wang T et al.: Hemodynamic forces sculpt developing heart valves through a KLF2-WNT9B paracrine signaling axis. Dev Cell 2017, 43:274-289 e275.

45. Cha B, Geng X, Mahamud MR, Fu J, Mukherjee A, Kim Y, Jho EH, Kim TH, Kahn ML, Xia L et al.: Mechanotransduction activates canonical Wnt/beta-catenin signaling to promote lymphatic vascular patterning and the development of lymphatic and lymphovenous valves. Genes Dev 2016, 30:1454-1469.

46. Chan CJ, Heisenberg CP, Hiiragi T: Coordination of morphogenesis and cell-fate specification in development. Curr Biol 2017, 27:R1024-R1035.

47. Fernandez-Sanchez ME, Serman F, Ahmadi P, Farge E: Mechanical induction in embryonic development and tumor growth integrative cues through molecular to multicellular interplay and evolutionary perspectives. Methods Cell Biol 2010, 98:295-321.

48. Monier B, Pelissier-Monier A, Brand AH, Sanson B: An actomyosin-based barrier inhibits cell mixing at compartmental boundaries in Drosophila embryos. Nat Cell Biol 2010, 12:60-65 sup pp 61-69.

49. Aliee M, Roper JC, Landsberg KP, Pentzold C, Widmann TJ, Julicher F, Dahmann C: Physical mechanisms shaping the Drosophila dorsoventral compartment boundary. Curr Biol 2012, 22:967-976. 